\title{
Caracterização do lodo gerado no tratamento de lixiviado de aterro sanitário com coagulante à base de tanino
}

\section{Characterization of sludge generated from the treatment of sanitary landfill leachate with tannin-based coagulant}

\author{
Lucila Akiko Nagashima'; Carlos de Barros Júnior²; Amanda Shizuka Fujimura3; \\ Taluana Delakis Recanello ${ }^{3}$
}

Resumo

O presente trabalho teve como objetivo efetuar a caracterização do lodo resultante do tratamento de lixiviado gerado no aterro sanitário com o coagulante à base de tanino, denominado Tanfloc $\mathrm{SG}^{\circledR}$ cedido pela empresa TANAC SA. O tratamento do efluente foi desenvolvido em $\mathrm{pH} 7,4$ e [Tanfloc SG] $=$ $2700 \mathrm{mg} / \mathrm{L}$, condições consideradas ótimas na remoção de DQO e da turbidez. Realizaram-se análises químicas de fracionamento de metais por meio de extrações simples e de extrações sequenciadas, cujo resultado indicou que o resíduo sólido é constituído de $29 \%$ de óxido amorfo, $28 \%$ de resíduo final, $22 \%$ de óxido cristalino e $21 \%$ de fração trocável. Os ensaios de lixiviação mostraram que o lodo originado do tratamento com Tanfloc $\mathrm{SG}^{\circledR}$ é classificado como resíduo Classe I - perigoso, segundo a legislação brasileira (NBR 10.004/2004 - ABNT), indicando que tais resíduos necessitam de tratamento e devem ter como destino final os aterros industriais, por apresentarem periculosidade em função de sua toxicidade.

Palavras-chave: Lodo. Aterro sanitário. Extração seqüencial. Metais pesados.

\begin{abstract}
The aim of this study was to characterise the sludge resulting from the leachate treatment generated at sanitary landfill with tannin-based coagulant, denominated Tanfloc $\mathrm{SG}^{\circledR}$ supplied by TANAC SA Company. The effluent treatment was performed at pH 7.4 and [Tanfloc SG] $=2700 \mathrm{mg} / \mathrm{L}$, considered as optimum condition in COD and turbidity removal, however. Metal fractionation chemical analyses were done through simple and sequential extractions, with results indicating the solid residue is constituted by $29 \%$ of amorphous oxide, $28 \%$ of final residue, $22 \%$ of crystalline oxide and $21 \%$ of exchangeable fraction. The leaching tests have shown the sludge originated by Tanfloc $\mathrm{SG}^{\circledR}$ treatment is classified as Class I residue - considered dangerous, according to Brazilian legislation (NBR 10.004/2004 - ABNT), indicating that such residues need to be treated and must be disposed in industrial landfills, for being a hazardous material due to its toxicity.
\end{abstract}

Key-words: Sludge. Landfill sanitary. Sequential extraction. Heavy metals.

${ }^{1}$ Graduada em Química pela Universidade Estadual de Maringá e em Ciências de Primeiro Grau pela Faculdade Estadual de Educação, Ciências e Letras de Paranavaí (FAFIPA/UNESPAR), com mestrado (2004) e doutorado (2009) em Engenharia Química pela Universidade Estadual de Maringá. E-mail: lucilanagashima@uol.com.br.

${ }^{2}$ Doutor em Engenharia Química e professor do Departamento de Engenharia Química da Universidade Estadual de Maringá.

${ }^{3}$ Acadêmicas do Curso de Engenharia Química - Universidade Estadual de Maringá. Bolsistas do PIBIC - Fundação Araucária. 


\section{Introdução}

Olixiviadoéumamatrizdeextremacomplexidade, apresentando em sua composição elevados teores de compostos orgânicos e inorgânicos, nas formas dissolvida e coloidal, resultante da decomposição química e microbiológica dos resíduos depositados em um aterro (CHRISTENSEN et al., 2001; EL FADEL et al., 2002; MORAIS, 2005). No seu tratamento, o processo de coagulação/floculação tem como finalidade transformar material solubilizado, partículas em suspensão fina ou em estado coloidal, em agregados maiores. A remoção destes agregados ou flocos é responsável pela clarificação do lixiviado resultando no acúmulo de lodo. Tal resíduo apresenta materiais inorgânicos e orgânicos e constituintes resultantes dos compostos químicos aplicados no tratamento do lixiviado. Desta forma, assim como o lixiviado gerado no aterro sanitário, os lodos gerados pelo tratamento devem estar de acordo com os padrões e normas que regulamentam a disposição de resíduos.

O tratamento do lixiviado com o coagulante tanino é eficaz na remoção de poluentes orgânicos e inorgânicos (MORAES et al., 2007), porém o lodo gerado é impregnado de metais (NAGASHIMA, 2009), formando diversas frações cujos componentes podem ser identificados pela especiação química.

A análise química do teor de metais pesados no lodo gerado é insuficiente para um diagnóstico do impacto que estes elementos podem causar ao meio ambiente e muito menos para se definirem os níveis de risco. Para melhor avaliar o efeito dos metais no ambiente, é necessário o conhecimento da forma química em que estes se encontram conforme discutido por Petruzzelli, Lubrano e Guidi (1985). A análise química do lodo em termos de metais pesados tem sido avaliada por meio de extrações simples e extrações sequenciadas, que identificam as formas químicas nas quais esses elementos se encontram associados (AMARAL SOBRINHO et al., 1997).

Pela simplicidade e rapidez na determinação, diversos estudos testaram a viabilidade de extrações químicas para avaliar a biodisponibilidade de metais pesados nos solos contaminados e em lodos por extração fracionada (RIBEIRO FILHO et al., 1999; PIERZYNASKI; SCHAWAB, 1993).

O emprego de extrações sequenciadas, visando o fracionamento químico, demanda maior tempo nas análises, mas permitem inferências sobre a origem, forma de ocorrência, biodisponibilidade, fluxos e mobilidade dos metais (TESSIER; CAMPBELL; BISSON, 1979; RIBEIRO FILHO et al., 1999; DAS et al., 1999). Nessas extrações, uma mesma amostra é submetida a uma série de extrações contínuas, em frações definidas, cujo poder de extração aumenta a cada fração. $O$ extrator atua modificando as propriedades químicas, que influem na interação do metal com a fase sólida, promovendo a sua solubilização para que possa ser dosado por um método analítico conveniente. Assim, nessas extrações, pode-se observar a presença de metais em formas químicas mais lábeis, solúveis, trocáveis e associadas a carbonato, ou mais estáveis e de menor mobilidade e/ou, biodisponibilidade, ligados a óxidos de $\mathrm{Fe}$ e de $\mathrm{Mn}$, ligada à matéria orgânica $\mathrm{e}$ residual do lodo ou solo (TESSIER; CAMPBELL; BISSON, 1982; URE et al., 1993; RAMOS; HERNADEZ; GONZALEZ, 1994).

As extrações sequenciais têm sido aplicadas no fracionamento de metais pesados em lodo de esgoto (STOVER et al., 1976 apud AMARAL SOBRINHO et al., 1997), lodo de tratamento de água (FUNTES et al., 2004), em solos incubados com lodo de esgoto (CHANG et al., 1984), em solos contaminados por sucatas de baterias (WOWK, 2003), em solos tratados contaminados com metais pesados (GIBSON; FARMER, 1986 apud AMARAL SOBRINHO et al., 1997; RIBEIRO FILHO et al., 1999), em solos das indústrias (MILLER; McFREE, 1983; BIELICKA; BOJANOWSKA; WISNIEWSKI, 2005), em sedimentos (TESSIER; CAMPBELL; BISSON, 1979, 1982; TACK; VERLOO, 1995; OLIVIER; SILVA; MOTTA, 2007) e em solos provenientes de aterros sanitários (OYGARD; GJENGEDAL; MOBBS, 2008). 
Como ressaltado por CHLOPECKA et al. (1996), apesar das limitações e falta de métodos eficazes para determinar a biodisponibilidade dos metais, o método de Tessier, Campbell e Bisson (1979) é uma abordagem analítica adequada para se inferir sobre o comportamento dos metais no solo. Segundo Egreja (2000), Gomes em 1999 iniciou uma adaptação da metodologia que se ajusta melhor aos solos brasileiros gerando uma marcha em solos e sedimentos altamente intemperizados. Atividades semelhantes também foram efetuadas pelo Instituto Agronômico de Campinas, descrevendo os métodos de análise dos cátions e ânions por meio da difração dos Raios-X. Segundo Oliveira (2007), com a finalidade de padronizar os métodos de extração sequencial (procedimentos) e os reagentes, possibilitando comparações, o Bureau of European Commission (BEC) propôs uma marcha analítica com quatro etapas, cujo método é composto pelas seguintes frações: solúvel em ácido (ácido acético, $0,11 \mathrm{~mol} / \mathrm{L}, 16 \mathrm{~h}$ ), redutível (oxalato ácido de amônio $0,1 \mathrm{~mol} / \mathrm{L}, \mathrm{pH}=2,16 \mathrm{~h})$, oxidável $\left(\mathrm{H}_{2} \mathrm{O}_{2}\right)$ e residual $\left(\mathrm{HCl} / \mathrm{HF} / \mathrm{HNO}_{3}\right)$.

Este trabalho avaliou os teores e formas de metais presentes no lodo oriundo do tratamento do lixiviado (aterro sanitário) com coagulante à base de tanino por meio da especiação química e os resultados obtidos foram avaliados por indicadores estabelecidos na Norma Brasileira de Referência (NBR) 10.004/2004, da Associação Brasileira de Normas Técnicas (2004a ABNT).

\section{Material e métodos}

\section{Amostragem}

A amostra de lixiviado usada no experimento de coagulação/floculação com Tanfloc $\mathrm{SG}^{\circledR}$ foi coletada no aterro sanitário da cidade de Paranavaí, Estado do Paraná (Brasil) e a pesquisa foi desenvolvida a partir de outubro de 2007 a setembro de 2008, no laboratório de Controle de Poluição Ambiental da UniversidadeEstadual deMaringá, Estadodo Paraná. Para o tratamento do lixiviado foram empregados os reagentes hidróxido de sódio $(\mathrm{NaOH})$ e ácido clorídrico $(\mathrm{HCl})$ para ajuste do $\mathrm{pH}$ e tanino cationico Tanfloc $\mathrm{SG}^{\circledR}$, fornecido pela empresa TANAC S.A, como agente coagulante. As características iniciais do lixiviado estão apresentadas na Tabela 1 .

Tabela 1 - Características do efluente utilizado no tratamento com Tanfloc SG ${ }^{\circledR}$

\begin{tabular}{cccccc}
\hline Parâmetros & unidade & lixiviado bruto & Parâmetros & unidade & lixiviado bruto \\
\hline Temperatura & ${ }^{\circ} \mathrm{C}$ & 23,00 & $\mathrm{Al}$ & $\mathrm{mg} / \mathrm{L}$ & 26,13 \\
$\mathrm{pH}$ & - & 8,00 & $\mathrm{As}$ & $\mathrm{mg} / \mathrm{L}$ & 2,80 \\
Turbidez & $\mathrm{UNT}$ & 141,17 & $\mathrm{Ba}$ & $\mathrm{mg} / \mathrm{L}$ & 17,19 \\
Cor real & $\mathrm{mg} \mathrm{Pt} / \mathrm{L}$ & 4621,12 & $\mathrm{Cd}$ & $\mathrm{mg} / \mathrm{L}$ & 0,07 \\
Cor aparente & $\mathrm{mg} \mathrm{Pt} / \mathrm{L}$ & 6380,05 & $\mathrm{Ca}$ & $\mathrm{mg} / \mathrm{L}$ & 31,18 \\
Alcalinidade & $\mathrm{mg} \mathrm{CaCO}_{3} / \mathrm{L}$ & 1529,78 & $\mathrm{~Pb}$ & $\mathrm{mg} / \mathrm{L}$ & 0,70 \\
DQO & $\mathrm{mg} \mathrm{O}_{2} / \mathrm{L}$ & 2708,10 & $\mathrm{Cr}$ & $\mathrm{mg} / \mathrm{L}$ & 1,70 \\
DBO & $\mathrm{mg} \mathrm{O}_{2} / \mathrm{L}$ & 731,18 & $\mathrm{Co}$ & $\mathrm{mg} / \mathrm{L}$ & 2,10 \\
Cloreto & $\mathrm{mg} / \mathrm{L}^{\mathrm{m}}$ & 14,17 & $\mathrm{Cu}$ & $\mathrm{mg} / \mathrm{L}$ & 0,91 \\
P-total & $\mathrm{mg} / \mathrm{L}$ & 18,31 & $\mathrm{Fe}$ & $\mathrm{mg} / \mathrm{L}$ & 78,00 \\
N-amoniacal & $\mathrm{mg} / \mathrm{L}$ & 890,00 & $\mathrm{Mn}$ & $\mathrm{mg} / \mathrm{L}$ & 6,01 \\
N-nitrato & $\mathrm{mg} / \mathrm{L}$ & 4,21 & $\mathrm{Hg}$ & $\mathrm{mg} / \mathrm{L}$ & 0,09 \\
N-nitrito & $\mathrm{mg} / \mathrm{L}$ & 61,08 & $\mathrm{Ni}$ & $\mathrm{mg} / \mathrm{L}$ & 2,00 \\
Sólidos suspen- & $\mathrm{mg} / \mathrm{L}$ & 990,00 & $\mathrm{Ag}$ & $\mathrm{mg} / \mathrm{L}$ & 0,16 \\
sos totais & & & &
\end{tabular}




\begin{tabular}{lccccc}
$\begin{array}{c}\text { Sólidos dissol- } \\
\text { vidos totais }\end{array}$ & $\mathrm{mg} / \mathrm{L}$ & 3212,00 & $\mathrm{Na}$ & $\mathrm{mg} / \mathrm{L}$ & 93,78 \\
Sulfeto total & $\mathrm{mg} / \mathrm{L}$ & 2,38 & $\mathrm{Se}$ & $\mathrm{mg} / \mathrm{L}$ & 2,51 \\
Sulfato total & $\mathrm{mg} / \mathrm{L}$ & 41,39 & $\mathrm{Zn}$ & $\mathrm{mg} / \mathrm{L}$ & 6,70 \\
\hline
\end{tabular}

\section{Procedimento experimental}

Os experimentos de coagulação/floculação com Tanfloc $\mathrm{SG}^{\circledR}$ foram conduzidos nas condições ótimas de operação, obtidas pela metodologia de superfície de respostas, cuja combinação é constituída de $\left[\right.$ Tanfloc $\left.\mathrm{SG}^{\circledR}\right]=2700 \mathrm{mg} / \mathrm{L}$ e pH 7,4 (NAGASHIMA, 2009). Os resíduos sólidos obtidos no tratamento foram divididos em três partes (Figura 1): as duas primeiras porções foram transferidas para frascos âmbar e preservadas a $4^{\circ} \mathrm{C}$ para os testes de lixiviação e digestão total para a determinação dos metais (Ag, Al, As, Ba, Cd, Ca, Cr, Co, Cu, Fe, $\mathrm{Mn}, \mathrm{Hg}, \mathrm{Na}, \mathrm{Ni}, \mathrm{Pb}$, Se e $\mathrm{Zn}$ ). A última porção foi filtrada a vácuo, lavada com água deionizada para eliminar o excesso de $\mathrm{NaOH}$ e então, seca a $105^{\circ} \mathrm{C}$. Após esta etapa os sólidos foram removidos do filtro, moídos e homogeneizados, e armazenados para fracionamento químico e análises de difração de Raio-X.

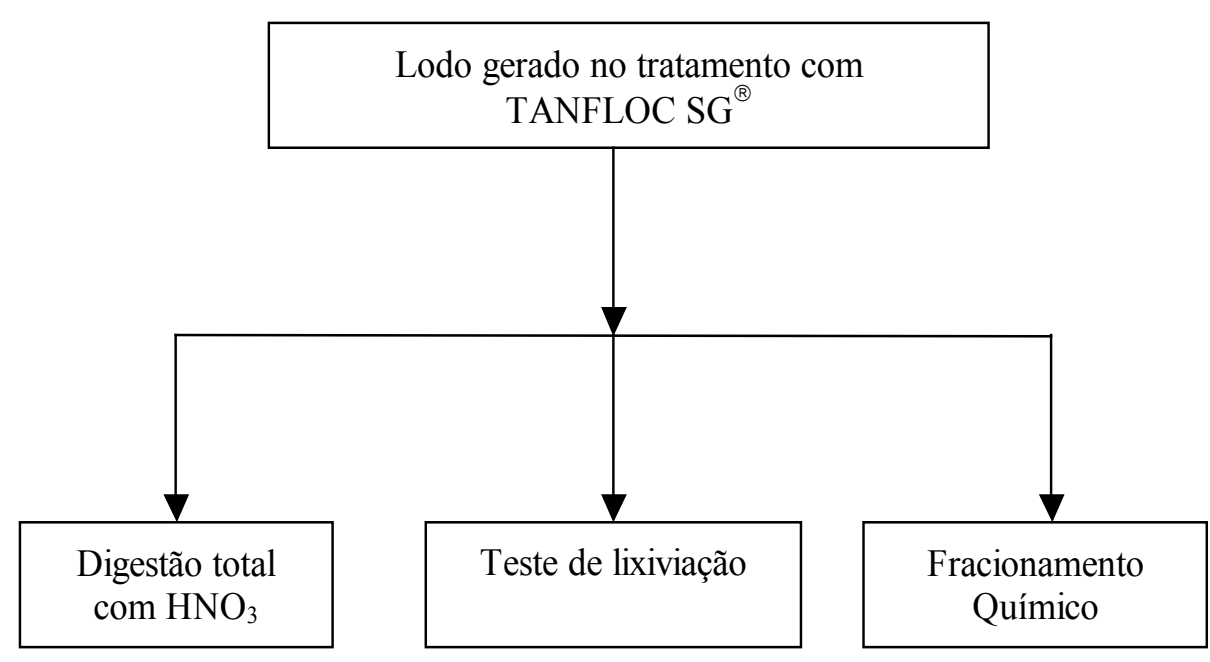

Figura 1. Esquema da caracterização do lodo

O lodo foi digerido com $\mathrm{HNO}_{3}$ concentrado e as amostras foram pré-concentradas, através do aquecimento em chapa elétrica, à temperatura de $60^{\circ} \mathrm{C}$, a fim de assegurar uma concentração metálica suficiente para a determinação, em função do limite de detecção imposto pela técnica de Espectrometria de Absorção Atômica de Chama. Os ensaios foram executados de acordo com as recomendações de Standard Methods for the Examination of Water and
Wastewater - APHA (1998), em duplicata.

Os testes de lixiviação seguiram a metodologia da norma NBR 10.005/2004 (ABNT, 2004b) que trata dos procedimentos para obtenção de extrato lixiviado de resíduos sólidos. Nesse processo, $100 \mathrm{~g}$ da massa seca foram misturados a $1600 \mathrm{~mL}$ de água desionizada, agitada vigorosamente por 5 minutos com agitador magnético e o $\mathrm{pH}$ foi determinado. 
Se esse for $\leq 5,0$, utilizar a solução de extração 1 (preparada com $5,7 \mathrm{~mL}$ de ácido acético glacial adicionada à água desionizada, $64,3 \mathrm{~mL}$ de $\mathrm{NaOH}$ $1,0 \mathrm{M}$ e completado o volume a $1 \mathrm{~L})$. Se o $\mathrm{pH}$ da mistura agitada for $>5,0$, adiciona-se $3,5 \mathrm{~mL}$ de $\mathrm{HCl}$ $1 \mathrm{~N}$, homogeneizando a solução e aquecendo a $50 \mathrm{oC}$ durante 10 minutos. Após o resfriamento da solução, medir novamente o $\mathrm{pH}$. Se o valor for $\leq 5,0$, utilizar a solução de extração 1 anteriormente descrita e se o valor de $\mathrm{pH}$ for $>5,0$, empregar a solução de extração 2 (preparada adicionando $5,7 \mathrm{~mL}$ de ácido acético glacial à água desionizada e completando o volume a 1L). A mistura, então, foi agitada por 24 horas em um agitador rotatório de frasco e posteriormente filtrada em membrana de fibra de vidro de $0,45 \mu \mathrm{m}$ de porosidade. Esse procedimento simula condições ácidas que favorecem a lixiviação de alguns contaminantes do lodo. Os extratos lixiviados foram então armazenados em frascos de vidro âmbar para a determinação dos metais $\mathrm{Ag}, \mathrm{Al}$, As, Ba, Ca, Cd, Co, Cr, Cu, Fe, Hg, Mn, Na, Ni, Pb, Se e Zn pela Espectrometria de Absorção Atômica.

Para o fracionamento químico foi empregado o método de extração sequencial que consiste na partição dos metais em quatro frações: trocável, óxido amorfo, óxido cristalino e residual, conforme a metodologia descrita e apresentada na Figura 2.

(a) Trocável - $1 \mathrm{~g}$ de amostra (lodo) foi submetida à extração com $8 \mathrm{~mL}$ de $\mathrm{MgCl}_{2}$, em pH 7, durante uma hora e com agitação contínua, em temperatura ambiente, segundo a metodologia proposta por Tessier, Campbell e Bisson (1979).

(b) Óxido amorfo - 0,5 g de amostra foi extraída com $100 \mathrm{~mL}$ de reagente de Tamm: $32,5 \mathrm{~g}$ de ácido oxálico $(\mathrm{COOH})_{2} \cdot 2 \mathrm{H}_{2} \mathrm{O}$ e $62,1 \mathrm{~g}$ de oxalato de amônio $\left(\mathrm{NH}_{4}\right)_{2} \mathrm{C}_{2} \mathrm{O}_{4} \cdot \mathrm{H}_{2} \mathrm{O}$ em 2,5 L de água deionizada; em $\mathrm{pH}$ 3,0 ajustada com $\mathrm{HCl}$ concentrado por 4 horas sob agitação constante, ao abrigo da luz e temperatura ambiente, de acordo com a metodologia proposta por Camargo et al. (1986).

(c) Óxido cristalino - 0,5 g de amostra foi adicionada a $40 \mathrm{~mL}$ de citrato de sódio $\left(\mathrm{Na}_{3} \mathrm{C}_{6} \mathrm{H}_{5} \mathrm{O}\right.$. $\left.{ }_{2} \mathrm{H}_{2} \mathrm{O}\right) \quad 0,3 \mathrm{M}$ e $5 \mathrm{~mL}$ de bicarbonato de sódio $\left(\mathrm{NaHCO}_{3}\right) 1 \mathrm{M}$ sob agitação e aquecimento até atingir a faixa de $75-50^{\circ} \mathrm{C}$ em banho-maria. Continuar o aquecimento por 1 hora com agitação esporádica. Repetir o tratamento com ditionito de sódio $\left(\mathrm{Na}_{2} \mathrm{~S}_{2} \mathrm{O}_{4}\right)$, esperando por mais trinta minutos, conforme a metodologia descrita por Mehra e Jackson (1958).

(d) Residual - 0,3 a 0,5 g de resíduo foi digerido numa mistura de ácido perclórico $\left(\mathrm{HClO}_{4}\right)$ e ácido nítrico $\left(\mathrm{HNO}_{3}\right)$ na proporção de 1:2.

Nos extratos obtidos pelo método de extração sequencial foram efetuadas as determinações dos metais Ag, Al, As, Ba, Ca, Cd, Co, Cr, Cu, Fe, Hg, $\mathrm{Mn}, \mathrm{Na}, \mathrm{Ni}, \mathrm{Pb}$, Se e Zn pela Espectrometria de Absorção Atômica.

As três primeiras etapas das extrações seletivas foram conduzidas em erlenmeyers de vidro de 250 $\mathrm{mL}$ e a agitação realizada com agitador magnético. A última etapa da extração foi efetivada em tubo Kjeldahl para minimizar perdas de material. Entre as extrações sucessivas, os resíduos foram lavados com respectiva solução extratora, separando-os dos sobrenadantes através da filtração a vácuo em membrana de éster de celulose (Millipore ${ }^{\circledR}$ de $0,45 \mu \mathrm{m})$. As soluções foram transferidas em balão volumétrico de $25 \mathrm{~mL}$ e seus volumes completados com água deionizada. A seguir, os extratos foram transferidos para vidro âmbar, acidificados para um $\mathrm{pH}<2$ com ácido nítrico $\mathrm{PA}\left(\mathrm{HNO}_{3}\right)$ e preservados a 4 oC para a determinação dos metais.

Os resíduos secos a $105^{\circ} \mathrm{C}$ em estufa por 12 horas foram resfriados em dessecador para a determinação da perda de massa. A seguir, os resíduos foram moídos e homogeneizados manualmente, novamente secos a $105^{\circ} \mathrm{C}$ para a remoção da umidade, resfriados novamente em dessecador e encaminhados para análise em difração de Raio-X (DRX). Essa análise foi efetuada em aparelho SHIMADZU D6000, no laboratório do Complexo de Centrais de Apoio à Pesquisa (COMCAP), utilizando radiação $\mathrm{CuK} \alpha$ e $\lambda=1,54178 \AA$, tensão $=40 \mathrm{kV}$, corrente $=40 \mathrm{~mA}$, 
varredura $2 \theta=5-70^{\circ}$, tamanho do passo $=0,020$, tempo do passo $=1 \mathrm{~s}$, velocidade de varredura $=$ $0,020 \mathrm{o} / \mathrm{s}$. A identificação das fases minerais nos difratogramas gerados foi efetuada a partir da posição e intensidades dos planos de difração. Os difratogramas da fase amorfa foram realizados por intermédio do difratometria diferencial de Raio-X (DDRX), a partir da subtração das intensidades dos difratogramas das amostras originais e dos resíduos obtidos após a extração da fase amorfa (BENATTI, 2005).

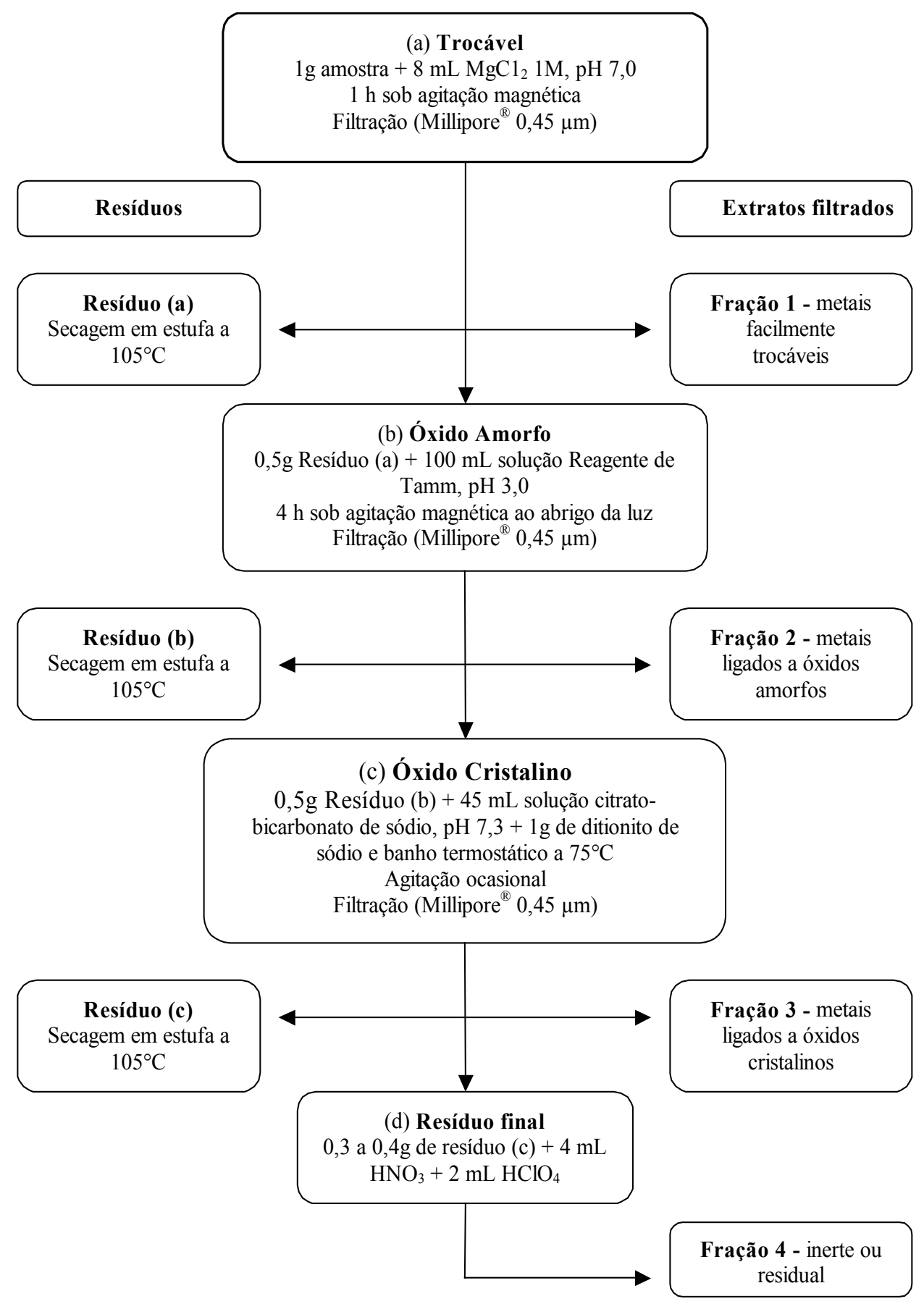

Figura 2. Esquema do método de extração sequencial aplicado aos resíduos obtidos com coagulante tanino (Tanfloc $\mathrm{SG}^{\circledR}$ ) 
$\mathrm{O} \mathrm{pH}$ foi determinado pelo método potenciométrico com auxílio de um medidor de $\mathrm{pH}$, marca DIGIMED DMPH-2, previamente calibrado com solução tampão de pH 4,0 e 7,0. A cor aparente e real, turbidez, sulfeto e sulfato, nitrato e nitrito foram determinados espectrofometricamente (Espectrofotômetro DR 2010, HACH). As análises de P-total, N-amoniacal, cloreto e alcalinidade forem efetuadas pelo método titulométrico, Demanda Bioquímica de Oxigênio (DBO), Demanda Química de Oxigênio (DQO) pelo método eletrométrico com membrana e sólidos suspensos e dissolvidos gravimetricamente, segundo as orientações do Standard Methods for the Examination of Water and Wastewater - APHA (1998).

Os elementos metálicos Ag, Al, As, Ba, Ca, Cd, $\mathrm{Co}, \mathrm{Cr}, \mathrm{Cu}, \mathrm{Fe}, \mathrm{Hg}, \mathrm{Mn}, \mathrm{Na}, \mathrm{Ni}, \mathrm{Pb}$, Se e $\mathrm{Zn}$ foram determinados pela Espectrometria de Absorção Atômica (Varian Spectra 50B).

\section{Resultados e discussão}

Os resultados das determinações de metais no resíduo sólido (lodo) mostraram que os elementos predominantes são $\mathrm{Na}, \mathrm{Fe}, \mathrm{Al}, \mathrm{Ba}$ e Ca (Tabela 2).
Tabela 2 - Concentração de metais presentes no lodo após a digestão total

\begin{tabular}{cc}
\hline Metais & Concentração $(\mathbf{m g} / \mathbf{L})$ \\
\hline $\mathrm{Ag}$ & 0,11 \\
$\mathrm{Al}$ & 20,11 \\
$\mathrm{As}$ & 1,60 \\
$\mathrm{Ba}$ & 22,22 \\
$\mathrm{Ca}$ & 12,00 \\
$\mathrm{Cd}$ & 0,05 \\
$\mathrm{Co}$ & 1,30 \\
$\mathrm{Cr}$ & 0,70 \\
$\mathrm{Cu}$ & 0,20 \\
$\mathrm{Fe}$ & 15,03 \\
$\mathrm{Hg}$ & 0,78 \\
$\mathrm{Mn}$ & 2,55 \\
$\mathrm{Na}$ & 60,00 \\
$\mathrm{Ni}$ & 0,17 \\
$\mathrm{~Pb}$ & 0,30 \\
$\mathrm{Se}$ & 1,00 \\
$\mathrm{Zn}$ & 2,00 \\
\hline
\end{tabular}

A Figura 3 mostra o difratograma de raio- $\mathrm{X}$ dos resíduos sólidos gerados após o tratamento com

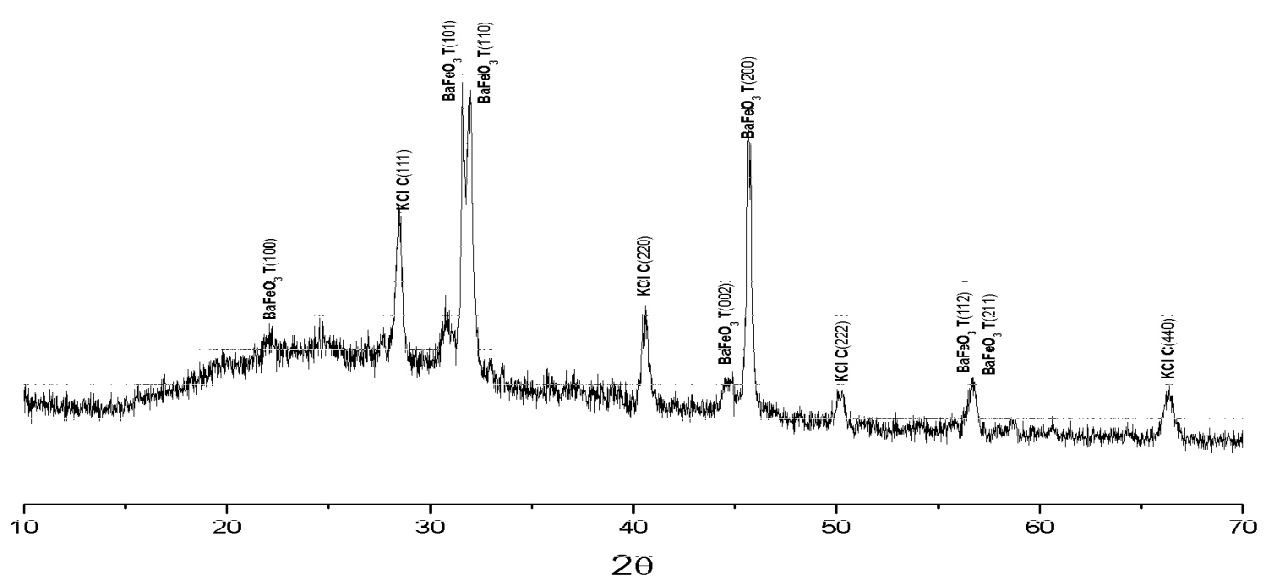

Figura 3 - Difratograma de raio-X do resíduo gerado no tratamento com Tanfloc $\mathrm{SG}^{\circledR}$ 
Tanfloc $\mathrm{SG}^{\circledR}$. Observa-se nesta figura que a fase amorfa é predominante no material, com a presença de $\mathrm{BaFeO}_{3}$ (óxido de ferrro e bário) e $\mathrm{KCl}$ (cloreto de potássio) de estruturas tetragonal e cúbica, respectivamente. As presenças de $\mathrm{Ba}$ e $\mathrm{Fe}$ foram, também, observadas em maiores concentrações na análise química total do resíduo, apresentadas na Tabela 2.

Para adequar cada vez mais a disposição do lodo às legislações ambientais, torna-se necessário o conhecimento da solubilidade destes metais, que é governado pela forma de associação destes no resíduo (BENATTI, 2005). A extração sequencial de elementos traços em resíduos permite determinar a solubilidade dos metais no resíduo que está relacionada à sua extração, decrescendo na ordem de sequência da extração. $\mathrm{O}$ extrator atua modificando as propriedades químicas, que influem fortemente na interação do metal com a fase sólida, promovendo a sua solubilização para que possa ser dosado por um método analítico conveniente (OLIVEIRA, 2007). Logo, amostras com maior teor de metais na Fração 1 (trocável) serão potencialmente mais perigosas do que aquelas apresentando um menor teor desta fração. Os metais extraídos na Fração 1 (trocável) correspondem àqueles adsorvidos fracamente, em particular aos retidos na superfície do resíduo com uma reduzida interação eletrostática. Na Fração 2 foram extraídos os metais ligados aos óxidos amorfos, que correspondem à parte reativa dos compostos do resíduo. Na Fração 3, os metais ligados a óxidos cristalinos foram extraídos, o que favorece a orientação preferencial dos minerais remanescentes ao serem submetidos à análise de difratometria de raio-X (BENATTI, 2005) e os minerais nesta fase estão presos por ligações extremamente fortes que não são rompidas pelas atividades metabólicas, tanto por plantas como por animais (OLIVEIRA, 2007). A Fração 4 contém minerais primários e secundários, capazes de reter em sua estrutura cristalina metais oriundos do efluente. Não há indicativo de que estes sejam liberados em um espaço de tempo razoável nas condições normais da natureza e são quimicamente estáveis e biologicamente inativos (BENATTI, 2005).

A Figura 4 representa o fracionamento $(\% \mathrm{em}$ massa) do lodo. Verifica-se que no resíduo oriundo do Tanfloc $\mathrm{SG}^{\circledR}$ há praticamente uma igualdade entre as frações, que é constituída de $29 \%$ de óxido amorfo, $28 \%$ de resíduo inerte, $22 \%$ de óxido cristalino e $21 \%$ de fração trocável.

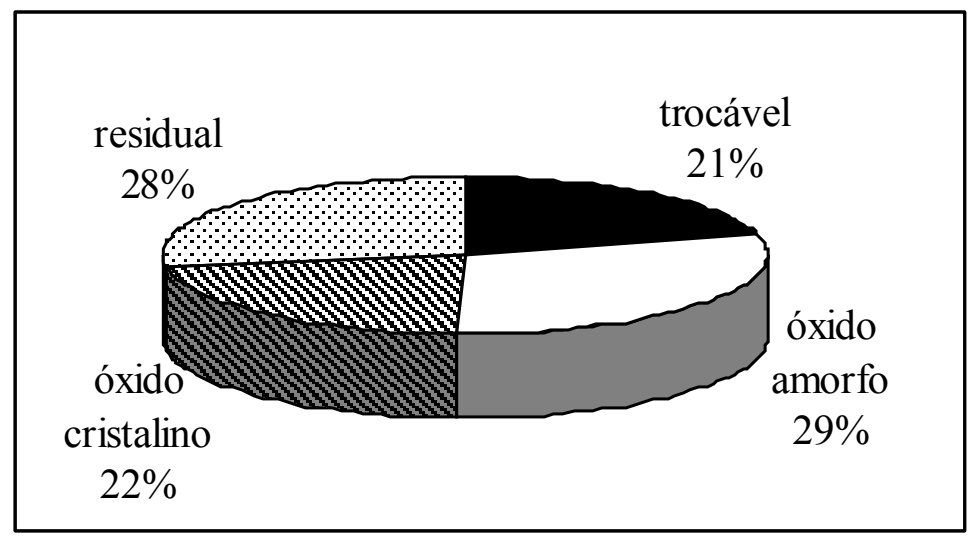

Figura 4 - Fracionamento (\% em massa) do resíduo sólido

A Tabela 3 apresenta os valores de concentração dos metais nas diversas frações dos resíduos estudados e a Figura 5 apresenta os valores, em percentagem, dos metais presentes nas quatro frações do extrato. 
Tabela 3 - Concentração $(\mathrm{mg} / \mathrm{kg})$ de metais nas diferentes frações do resíduo analisado

\begin{tabular}{|c|c|c|c|c|}
\hline \multirow[b]{2}{*}{ Metal } & \multicolumn{4}{|c|}{ Concentração (mg/L) } \\
\hline & trocável & óxido amorfo & óxido cristalino & residual \\
\hline $\mathrm{Ag}$ & 160 & nd & 462 & nd \\
\hline $\mathrm{Al}$ & 3133 & 8832 & 6266 & 21200 \\
\hline As & 900 & nd & 600 & nd \\
\hline $\mathrm{Ba}$ & 1584 & 1888 & 2036 & 915 \\
\hline $\mathrm{Ca}$ & 10540 & 266 & 662 & 1215 \\
\hline $\mathrm{Cd}$ & 29 & nd & 80 & nd \\
\hline Co & 500 & nd & 800 & 62,5 \\
\hline $\mathrm{Cr}$ & 130 & nd & 410 & 275 \\
\hline $\mathrm{Cu}$ & 110 & 18 & 124 & 80 \\
\hline $\mathrm{Fe}$ & 19780 & 36180 & 15380 & 5662,5 \\
\hline $\mathrm{Hg}$ & 30 & nd & 80 & nd \\
\hline $\mathrm{Mn}$ & 2110 & - & nd & nd \\
\hline $\mathrm{Na}$ & 14030 & 28428 & 28980 & 34500 \\
\hline $\mathrm{Ni}$ & 30 & nd & 270 & nd \\
\hline $\mathrm{Pb}$ & 20 & nd & 20 & 50 \\
\hline $\mathrm{Se}$ & nd & nd & nd & nd \\
\hline $\mathrm{Zn}$ & 455 & 620 & 288 & 7175 \\
\hline
\end{tabular}

nd = não detectado

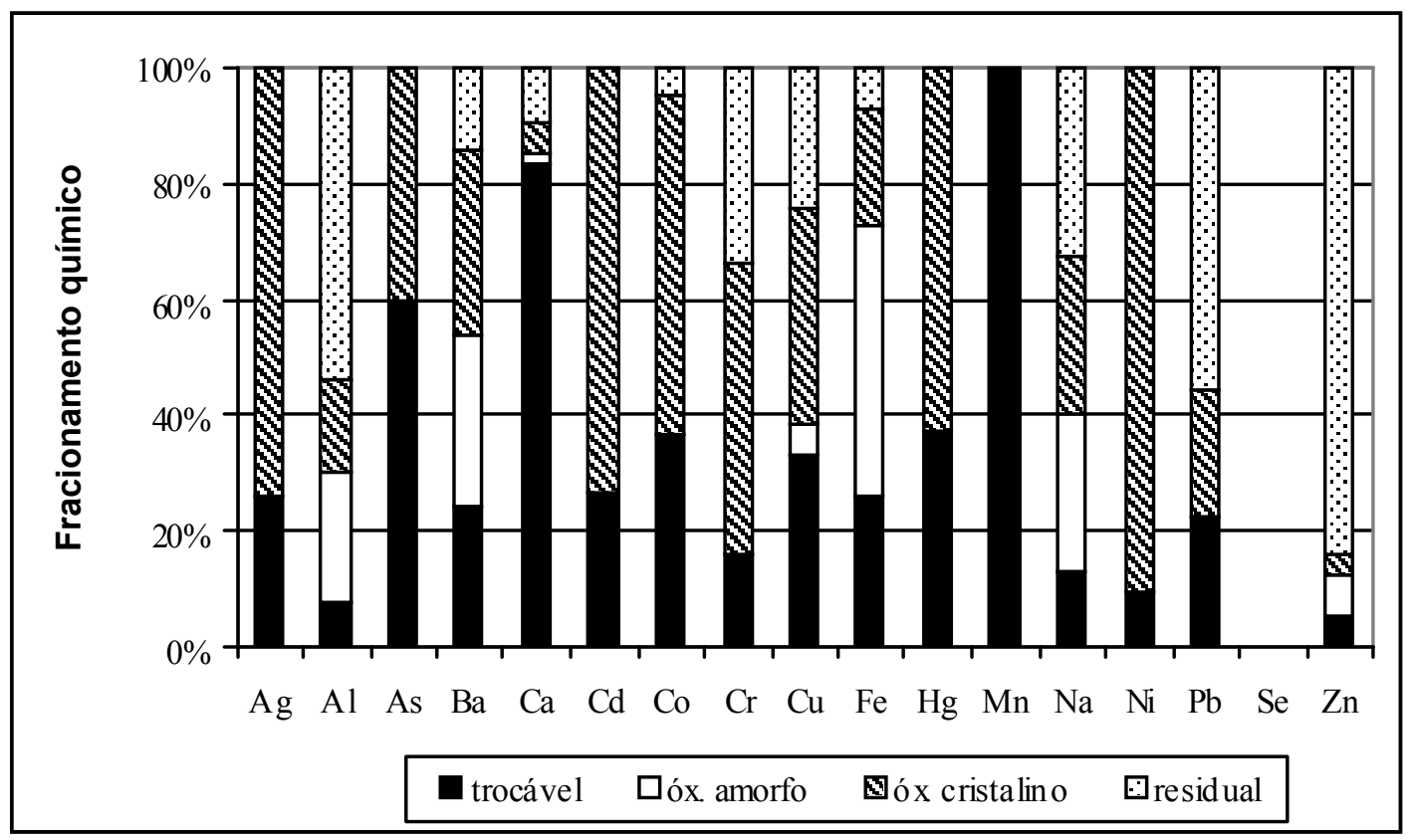

Figura 5. Fracionamento químico do resíduo do tratamento com Tanfloc $\mathrm{SG}^{\circledR}$ 
Todos os metais analisados (exceto $\mathrm{Se}$ ) foram detectados na Fração 1 (Figura 5). Estes metais estariam associados a sais solúveis, ou simplesmente adsorvidos na superfície do resíduo. O Mn se apresenta totalmente na forma trocável, os metais As e Ca aparecem com valores superiores a 50\% do teor total nesta forma (trocável) evidenciando o alto potencial de biodisponibilidade desses elementos no ambiente. Além disso, a Figura 5 permite observar o fracionamento químico de cada resíduo. O metal $\mathrm{Fe}$ encontra-se predominantemente na fase amorfa dos óxidos, porém os metais $\mathrm{Ag}, \mathrm{Cd}, \mathrm{Co}, \mathrm{Cr}, \mathrm{Hg}$ e Ni aparecem principalmente na forma de óxido cristalino. Elementos como Al, $\mathrm{Pb}$ e $\mathrm{Zn}$ encontram- se predominantemente na forma residual, no entanto, há metais que se apresentam nas quatro frações tais como Al, Ba, $\mathrm{Ca}, \mathrm{Cu}, \mathrm{Fe}, \mathrm{Na}$ e $\mathrm{Zn}$.

Os difratogramas obtidos na difração de raio-X de cada fração do resíduo estão representados na Figura 6. Estes difratogramas mostram que o reagente cloreto de magnésio $\left(\mathrm{MgCl}_{2}\right)$ extraiu o componente $\mathrm{KCl}$ da fração trocável, no entanto a utilização de reagentes como oxalato ácido de amônio e do ditionito na extração de material amorfo e cristalino, respectivamente, não foram capazes de reduzir o teor de Fe presentes em todas as frações do fracionamento químico (Figuras $6 \mathrm{a}, \mathrm{b}, \mathrm{c}$ ).

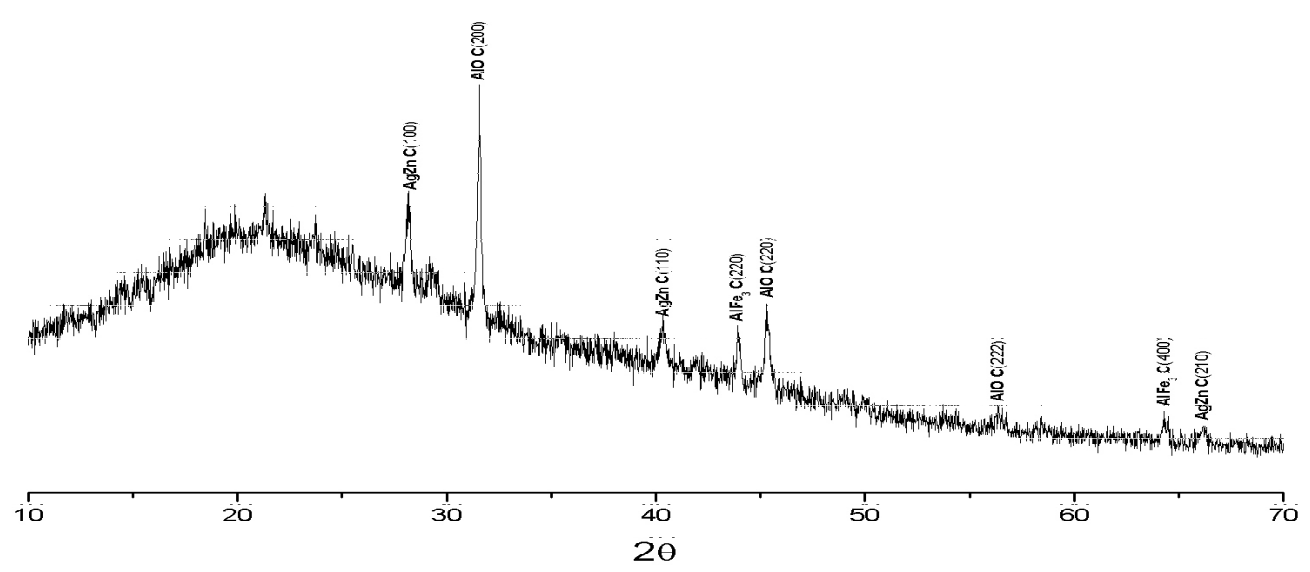

(a)

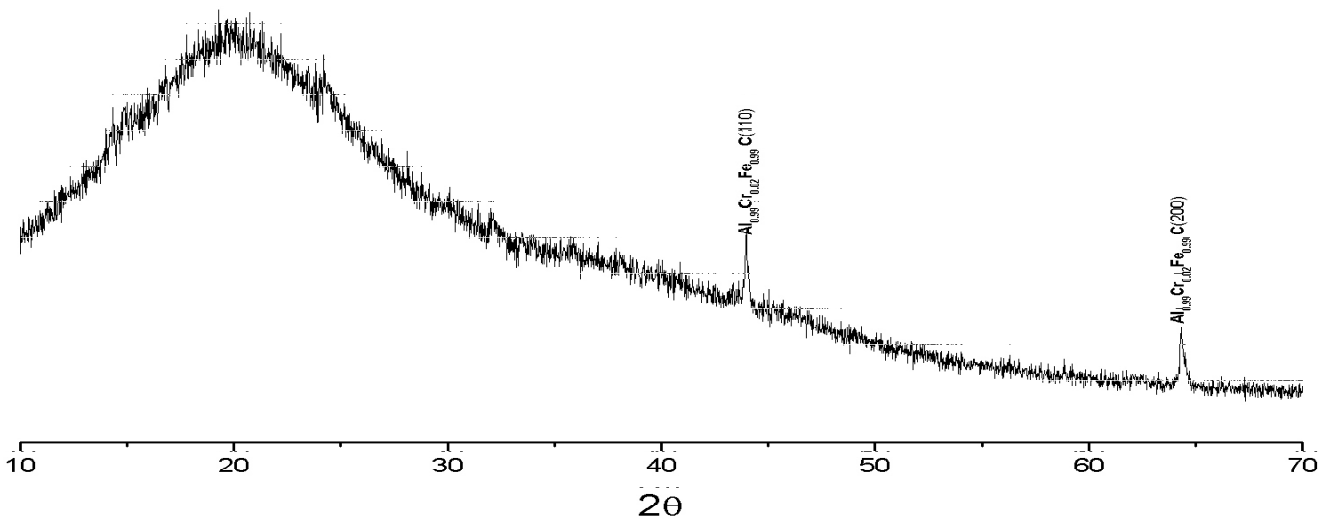

(b) 


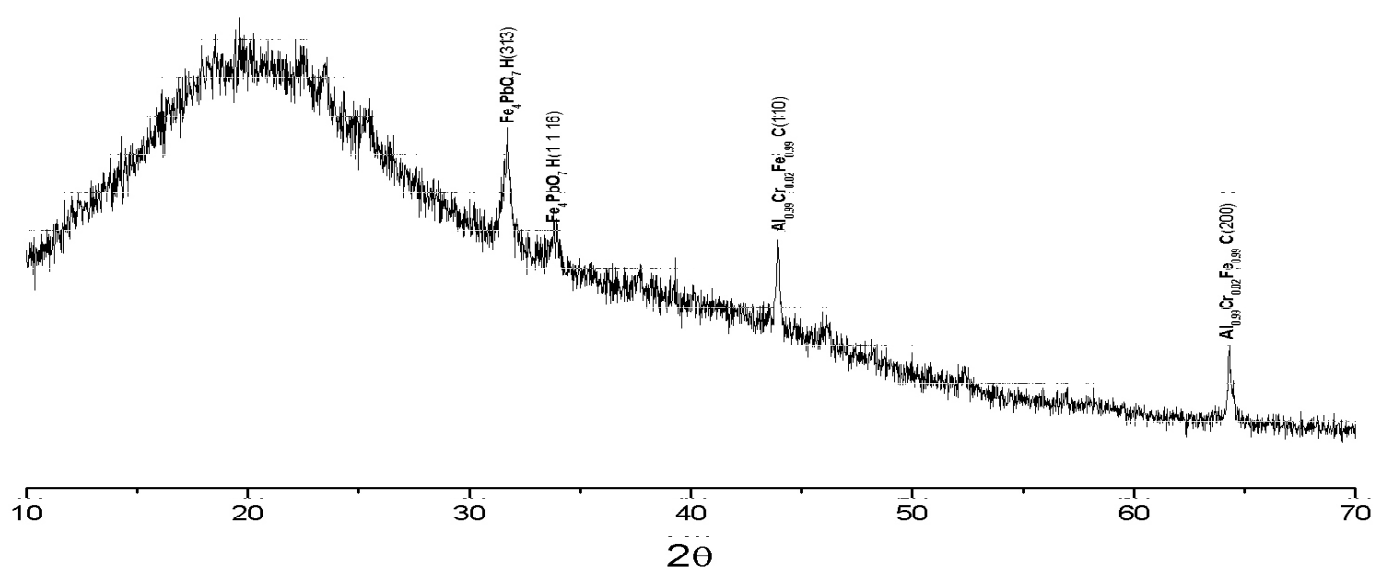

Figura 6. Difratogramas de raio-X das diferentes frações do resíduo oriundo do tratamento com Tanfloc $\mathrm{SG}^{\circledR}$ : (a) após a extração de material trocável; (b) após a extração de óxidos amorfos; (c) após a extração do óxido cristalino.

Estes difratogramas de raio-X indicam que o resíduo gerado com o tratamento Tanfloc $\mathrm{SG}^{\circledR}$ apresenta um perfil típico de material constituído pela fase amorfa, podendo-se identificar a presença de Ag, Zn, Al e Fe. Após a extração da fração amorfa, verifica-se a presença de material metálico $\mathrm{Al}, \mathrm{Fe}$ e $\mathrm{Cr}$, sendo que na fração residual ou inerte, o resíduo apresenta características de liga metálica formada pelos elementos $\mathrm{Al}, \mathrm{Cr}, \mathrm{Fe}$ e compostos à base de material ferroso e plumboso $\left(\mathrm{Fe}_{4} \mathrm{PbO}_{7}-\right.$ plumboferrita).

Verificou-se que os resíduos resultantes do tratamento apresentam em sua composição metais como $\mathrm{Hg}, \mathrm{Pb}, \mathrm{Cd}$ e As (Tabela 2), e que mesmo em condições ambientais não agressivas podem ocorrer a lixiviação e solubilização de metais a partir destes. Para se obter previsões corretas sobre os possíveis processos de mobilização dos elementos no ambiente, as amostras de lodo foram submetidas ao ensaio de lixiviação, cujos resultados se encontram na Tabela 4. Observou-se que os metais As e Hg oriundos do resíduo do tratamento com Tanfloc $\mathrm{SG}^{\circledR}$, ao serem submetidos ao teste de lixiviação, apresentaram concentrações que ultrapassaram os limites máximos estabelecidos pela NBR 10.004/2004 (ABNT, 2004a).

Tabela 4 - Resultados das análises químicas (mg/L) dos ensaios de lixiviação

\begin{tabular}{cccc}
\hline Metal & Código* & Concentração (mg/L) & Limite máximo* \\
\hline $\mathrm{Ag}$ & $\mathrm{D} 012$ & 0,05 & 5,00 \\
$\mathrm{Al}$ & $\mathrm{D} 005$ & 0,63 & 1,00 \\
$\mathrm{As}$ & - & 1,01 & 1,0 \\
$\mathrm{Ba}$ & $\mathrm{D} 006$ & 3,37 & 70,00 \\
$\mathrm{Ca}$ & - & 1,33 & - \\
$\mathrm{Cd}$ & $\mathrm{D} 007$ & nd & 0,50
\end{tabular}




\begin{tabular}{cccc}
$\mathrm{Co}$ & - & 0,03 & - \\
$\mathrm{Cr}$ & $\mathrm{D} 009$ & 0,04 & 5,00 \\
$\mathrm{Cu}$ & - & 0,04 & - \\
$\mathrm{Fe}$ & - & 0,17 & 0,10 \\
$\mathrm{Hg}$ & $\mathrm{D} 011$ & 0,11 & - \\
$\mathrm{Mn}$ & - & 1,71 & - \\
$\mathrm{Na}$ & - & 29,33 & - \\
$\mathrm{Ni}$ & - & 0,10 & 1,00 \\
$\mathrm{~Pb}$ & $\mathrm{D} 008$ & 0,37 & 1,00 \\
$\mathrm{Se}$ & $\mathrm{D} 013$ & 0,09 & - \\
$\mathrm{Zn}$ & - & 0,56 & \\
\hline
\end{tabular}

nd - não detectado

*Anexo F - NBR 10.004/2004 (ABNT, 2004a).

Baseando-se nos resultados analíticos químicos (teste de lixiviação), o resíduo foi caracterizado como resíduo de Classe I - perigoso por apresentar As e $\mathrm{Hg}$, no extrato lixiviado, em teores superiores àqueles admitidos pela norma NBR 10.004/2004.

Assim, esse resultado indica potencial de contaminação do solo e das águas, por metais se os resíduos obtidos após o tratamento com Tanfloc $\mathrm{SG}^{\circledR}$ forem dispostos inadequadamente. Tais resíduos necessitam de tratamento e devem ter como destino final os aterros industriais, por apresentarem periculosidade em função de sua toxicidade.

\section{Conclusão}

Após o tratamento do lixiviado pelo processo de coagulação/floculação com o coagulante Tanfloc $\mathrm{SG}^{\circledR}$ verificou-se a remoção de metais, especialmente dos elementos $\mathrm{Hg}, \mathrm{Al}$ e $\mathrm{Na}$. Entretanto, ainda é necessária a aplicação de um método adicional para a remoção dos metais remanescentes para atender aos padrões estabelecidos pela legislação em vigor para o descarte do efluente. Além disso, o tratamento do lixiviado com coagulante Tanfloc $\mathrm{SG}^{\circledR}$ gerou uma quantidade de resíduo sólido (lodo) ao final do processo. $\mathrm{O}$ fracionamento desses resíduos em massa mostrou é constituída de $29 \%$ de fração na forma de óxido amorfo, $28 \%$ na forma residual, $22 \%$ formando óxido cristalino e 21\% na forma trocável. Todos os elementos pesquisados apresentam a fase trocável (com exceção Se), que é a forma mais lábil, de maior mobilidade no meio ambiente.

As análises mostraram que os resíduos estão incrustados de metais removidos da porção líquida e os resultados obtidos a partir da caracterização destes resíduos mostraram que, mesmo em condições ambientais não agressivas, pode ocorrer a lixiviação de metais a partir destes. Os resíduos foram classificados, de acordo com a norma NBR 10.004/2004 da ABNT (2004a), como perigoso (Classe I), apresentando grande potencial de contaminação do solo, águas subterrâneas e superficiais por metais pesados como $\mathrm{As}$ e $\mathrm{Hg}$, se dispostos em locais não adequados, sendo necessária a implementação de um processo de estabilização antes da disposição destes no ambiente.

\section{Agradecimento}

À Fundação Araucária pelo apoio financeiro e ao complexo de Centrais de Apoio à Pesquisa (COMCAP) pela realização das análises de Difração de Raio-X.

\section{Referências}


AMARAL SOBRINHO, N. M. B.; VELlOSO, A. C. X.; COSTA, L. M.; OLIVEIRA, C. Formas químicas de zinco e sua absorção por plantas de milho cultivadas em solo tratado com resíduo siderúrgico. Revista Brasileira de Ciências do Solo, Campinas, v. 18, p. 313-320, 1997.

APHA. Standard methods for the examination of water and wastewater. 20th ed. Washington: American Public Health Association, 1998.

ASSOCIAÇÃO BRASILEIRA DE NORMAS TÉCNICAS - ABNT. NBR 10.004: resíduos sólidos classificação. Rio de Janeiro: ABNT, 2004a.

NBR 10.005: procedimentos para obtenção de extrato lixiviado de resíduos sólidos. Rio de Janeiro: ABNT, 2004b.

BENATTI, C. T. Caracterização e tratamento do efluente de laboratório por processos químicos. 2005. Tese (Doutorado em Engenharia Química) - Universidade Estadual de Maringá, Departamento de Engenharia Química, Maringá.

BIELICKA, A.; BOJANOWSKA, I.; WISNIEWSKI, A. Sequential extraction of chromium from galvanic waste sludge. Polish Journal of Environmental Studies, Olsztyn, v. 14, n. 2, p. 145-158, 2005.

CAMARGO, O. A.; MONIZ, A. C.; JORGE, J. A.; VALADARES, J. M. Métodos de análise química, mineralógica e física de solos do Instituto Agronômico de Campinas. Campinas: Instituto Agronômico, 1986. (BOLETIM TÉCNICO, nº 106).

CHANG, A. C.; PAGE, A. L.; WARNEKE, J. E.; GRUREVIC, E. Sequential extraction of soil heavy metals in sewage following a sludge application. Journal Environmental Quality, Madison, v.13, n. 1, p. 33-37, 1984.

CHLOPECKA, A.; BACON, J. R.; WILSON, M. J.; KAY, J. Forms of cadmium, lead and zinc in contaminated soils from Southwest Poland. Journal Environmental Quality, Madison, v. 25, p. 69-79, 1996.

CHRISTENSEN, T. H.; BJERG, P. P. L.; JENSEN, D. L.; CHRISTENSEN, A.; BAUM, A.; ALBRECHTSEN, H. J.; HERON, G. Biochemistry of landfill leachate plumes. Applied Environmental Geochemistry, London, v. 16, p. 659-718, 2001.

DAS, A. K.; CHAKRABORTY, R.; CERVERA, M. L., LA GUARDIA, M. Metal speciation in solid matrices. Talanta, London, v. 42, n. 8, p. 1007-1030, 1999.
EGREJA, F. B. F. Extração sequencial de metais pesados em solos altamente intemperizados: utilização de componentes modelo e planejamento com misturas ternárias na otimização do método. 2000. Tese (Doutorado em Ciência do Solo) - Universidade Federal de Viçosa, Viçosa.

EL FADEL, M.; DOUSEID, E.; CHAHINE, W.; ALAYLIC, B. Factors influencing solid waste generation and management. Waste Management, Amsterdam, v. 22, p. 269-276, 2002.

FUNTES, A.; LHORÉNS, M.; SÁEZ, J.; SOLER, A.; AGUILAR, M. I.; ORTUÑNO, J. F.; MESEGUER, V. F. Simple and sequential extraction of heavy metals from different sewage sludges. Chemosphere, Amsterdam, v. 54, n. 8, p. 1039-1047, 2004.

MEHRA, O. P.; JACKSON, M. L. Iron oxide removal from soils and clays by a dithionite-citrate system buffered with sodium bicarbonate. Clays and Clay Minerals, New York, v. 7, n. 1, p. 317-327, 1958.

MILlER, W. P.; McFREE, W. W. Distribution of cadmium, zinc, copper and lead in soils of industrial Northwestern Indiana. Journal of Environmental Quality, Madison, v. 12, p. 29-33, 1983.

MORAES, L. C. K.; BERGAMASCO, R.; TAVARES, C. R. G.; GIOVANI, M. C.; HENNIG, D. Avaliação da eficiência de remoção de cor e turbidez, utilizando como agente coagulante os taninos vegetais com a finalidade de obtenção de água tratada. In: CONGRESSO BRASILEIRA DE ENGENHARIA SANITÁRIA E AMBIENTAL, 24., 2007, Belo Horizonte. Anais... Belo Horizonte: ABES, 2007. CD-ROM.

MORAIS, J. L. Estudo da potencialidade de processos oxidativos avançados, isolados e integrados com processos biológicos tradicionais, para tratamento de chorume de aterro sanitário. 2005. Tese (Doutorado em Química) - Universidade Federal do Paraná, Curitiba.

NAGASHIMA, L. A. Monitoramento de lixiviado em lagoa de estabilização e estudo da aplicabilidade do Reagente Fenton e do coagulante tanino como formas de tratamento. 2009. Tese (Doutorado em Engenharia Química) - Universidade Estadual de Maringá, Maringá.

OLIVIER, S.; SILVA, V. L.; MOTTA, M. Emprego de planejamento fatorial no desenvolvimento de uma metodologia para extração de zinco de resíduos galvânicos. Química Nova, São Paulo, v. 30, n. 7, p. 1750-1753, 2007. 
OLIVEIRA, M. R. Investigação da contaminação por metais pesados da água e do sedimento da corrente nas margens do Rio São Francisco e tributários, a jusante da Represa da CEMIG, no município de Três Marias. 2007. Tese (Doutorado em Geologia) - Universidade Federal de Minas Gerais, Belo Horizonte.

OYGARD, J. K.; GJENGEDAL, E.; MOBBS, H. J. Trace element exposure in the environmental from MSW landfill leachate sediments measured by a sequential extraction technique. Journal of Hazardous Materials, Amsterdam, v. 153, n. 1/2, p. 751-758, 2008.

PETRUZZELLI, G.; LUBRANO, L.; GUIDI, G. Heavy metal extractability. Biocycle, Emmaus, v. 26, p. 46-49, 1985.

PIERZYNASKI, G. M.; SCHWAB, A. P. Bioavailability of zinc, cadmium and lead in a metal contaminated alluvial soil. Journal of Environmental Quality, Madison, v. 22, p. 247-254, 1993.

RAMOS, L.; HERNANDEZ, L. M.; GONZALEZ, M. J. Sequential fractionation of copper, lead, cadmium and zinc in soils from or near Noñana National Park. Journal Environmental Quality, Madison, v. 23, p. 50-57, 1994.

RIBEIRO FILHO, M. R.; CURI, N.; SIQUEIRA, J. O.; DA MOTTA, P. E. F. Metais pesados em solos de área de rejeitos de indústria de processamento de zinco. Revista Brasileira de Ciência do Solo, Campinas, v. 23, n. 2, p. 453-464, 1999.
TACK, F. M.; VERLOO, M. G. Chemical Speciation and fractionation in soil and sediment heavy metal analysis. International Journal of Environmental Analytical Chemistry, London, v. 59, n. 2/4, p. 225-238, 1995.

TESSIER, A.; CAMPBELL, P. G. C.; BISSON, M. Sequential extraction procedure for the speciation of particulate trace metals. Analytical Chemistry, Washington, v. 51, n. 7, p. 844-851, 1979.

Particulate trace metal speciation in stream sediments and relationships with grain size: implications for geochemical exploration. Journal of Geochemical Exploration, Amsterdam, v. 16, n. 2, p. 77-104, 1982.

URE, A. M.; QUEVAUVILlER, P. H.; MUNTAU, H.; GRIEPINK, B. Speciation of heavy metals in soils and sediments. An account of the improvement and harmonization of extraction techniques undertaken under the auspices of the BCR of the commission of the European Communities. International Journal Analytical Chemistry, London, v. 51, n. 1/4, p. 135-151, 1993.

WOWK, G. I. T. H. Avaliação da contaminação do solo por chumbo proveniente da reciclagem de sucatas de baterias em área de várzeas no município de Paula Freitas (PR). 2003. Dissertação (Mestrado em Ciências do Solo) - Universidade Federal do Paraná, Curitiba.

Recebido em 20 Agosto, 2009 - Received on August 20, 2009

Aceito em 18 Março, 2010 - Accepted on March 18, 2010. 